\title{
BMJ Open Effects of a school-based prevention programme on smoking in early adolescence: a 6-month follow-up of the 'Eigenständig werden' cluster randomised trial
}

\author{
Barbara Isensee, Julia Hansen, Karin Maruska, Reiner Hanewinkel
}

To cite: Isensee B, Hansen J, Maruska $\mathrm{K}$, et al. Effects of a school-based prevention programme on smoking in early adolescence: a 6-month follow-up of the 'Eigenständig werden' cluster randomised trial. BMJ Open 2014;4: e004422. doi:10.1136/ bmjopen-2013-004422

- Prepublication history for this paper is available online. To view these files please visit the journal online (http://dx.doi.org/10.1136/ bmjopen-2013-004422).

Received 7 November 2013 Revised 10 December 2013 Accepted 20 December 2013

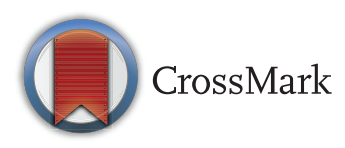

Institute for Therapy and Health Research (IFT-Nord), Kiel, Germany

Correspondence to Dr Barbara Isensee; isensee@ift-nord

\section{ABSTRACT}

Objectives: To test the effects of a school-based prevention programme on students' smoking-related behaviour, attitudes and knowledge 6 months after implementation over 2 school-years has ended.

Design: Two-arm prospective cluster randomised controlled trial with a follow-up survey 6 months after end of programme implementation, that is, 26 months after baseline.

Setting: 45 public secondary schools from four federal states in Germany (Bremen, Hesse, North Rhine-Westphalia, Schleswig-Holstein).

Participants: A total of 3444 students from 172 classes with a mean age of 10.37 years $(S D=0.59)$ and $47.9 \%$ girls at baseline. Analysis sample with follow-up up data merged to baseline data comprises 2513 datasets $(73 \%)$.

Intervention: 'Eigenständig werden $5+6$ ', a school-based prevention programme for grades 5 and 6 to enhance substance-specific and general life skills, consisting of 14 units (à $90 \mathrm{~min}$ ) and two workshops (4-6 h) being taught over a time period of 2 school-years by trained teachers.

Outcome measures: Lifetime and current smoking, incidence of smoking in baseline never smokers, smoking-related knowledge, attitudes, perceived norms of smoking and self-efficacy to refuse cigarette offers were assessed in students.

Results: 6 months after the end of programme implementation, students of intervention classes showed significantly lower rates for lifetime smoking (adjusted OR=0.63; 95\% $\mathrm{Cl} 0.41$ to $0.96 ; p=0.026$ ) and incidence of smoking (adjusted $\mathrm{OR}=0.66 ; 95 \% \mathrm{Cl} 0.43$ to 1.00; $p=0.047$ ), a higher increase of smoking-related knowledge (adjusted $\beta=9.38 ; 95 \% \mathrm{Cl} 6.73$ to 12.04 ; $p<0.001)$ and a greater change in attitudes towards a more critical perception of risks and disadvantages of smoking (adjusted $\beta=0.10 ; 95 \% \mathrm{Cl} 0.03$ to 0.16 ; $\mathrm{p}=0.002$ ). No group differences were found for current smoking, perceived norms of smoking and self-efficacy to refuse cigarette offers.

Conclusions: Participation in the school-based prevention programme 'Eigenständig werden $5+6$ ' may have small effects on smoking behaviour and
Strengths and limitations of this study

Findings were retrieved from a clusterrandomised controlled trial with a well-powered sample.

- Sophisticated statistical methods controlling for confounders and taking clustering of data into account were used.

- Dropout, reliance on self-reports and lack of control for treatment integrity may be limiting factors to this study.

attitudes and a moderate effect on smoking-related knowledge.

Trial registration: Current Controlled Trials ISRCTN99442407

\section{BACKGROUND}

Smoking remains to be the single greatest preventable cause of mortality worldwide, being a major risk factor for a number of life-threatening diseases, including various cancers, cardiovascular diseases and lung diseases. ${ }^{1}$ Although reductions in smoking rates in adolescents have been documented for many Western countries including Germany ${ }^{2}$ over the last decade, smoking prevention is still a main issue for public health. Most recent data from Germany reveal smoking rates of about $12 \%$ for female and male adolescents aged $12-17$ years and $6.6 \%$ for young adolescents aged $12-15$ years. $^{23}$

School programmes are often one of the first approaches mentioned in efforts to denormalise tobacco use and to raise awareness of tobacco's hazardous nature. Prominent approaches to prevent smoking in students are the development and improvement of general life skills, as well as the fostering of skills for resisting social influence 
and substance-specific skills in adolescence. General life skills are considered to empower adolescents in challenging situations and to help them to master life as competent as possible. ${ }^{4}$ By enhancing these skills, it is assumed to prevent substance use and abuse, since substance use is considered as a dysfunctional strategy to cope with everyday challenges and developmental tasks in adolescence. In line with the social influence approach, ${ }^{5}$ smoking is conceptualised as a result of influences emerging from the adolescent's environment such as peers, family or media by normative processes and/or overt cigarette offers. Therefore, correction of inaccurate norms is one important component of these programmes to adjust the often overestimated prevalence of smoking in adolescence towards more conservative and realistic norms. ${ }^{6}$ Skill training following these approaches is considered to 'inoculate' students against influences encouraging them to smoke, to help them resist temptations from peers to smoke and to correct normative expectations towards smoking.

For more than 40 years, prevention programmes have been implemented and evaluated within the school setting. However, the empirical evidence of the efficacy of these approaches is not yet uniquely convincing. ${ }^{7-12} \mathrm{~A}$ recent Cochrane review on the effects of school-based smoking prevention programmes ${ }^{13}$ selected randomised controlled trials where students, classes, schools or school districts were randomised to intervention $\operatorname{arm}(\mathrm{s})$ versus a control group, and followed-up for at least 6 months. One hundred and thirty-four studies involving 428293 participants met the inclusion criteria of the review, which revealed an overall significant intervention effect on the onset of smoking at the longest follow-up, while there was no overall effect at follow-ups at 1 year or less. When analysing intervention effects not only on smoking behaviour, but also on further outcomes addressed directly or indirectly by school-based interventions like attitudes towards smoking, resistance skills or smoking-related knowledge, findings might be condensed into a more or less consistent pattern with medium effects being found for knowledge and, if any, small effects for attitudes, skills and use. ${ }^{14-16}$

Among altogether 37 trials on smoking prevention strategies from the time period 2001-2006 which were included in a Health Technology Assessment, only one study originated from Germany. ${ }^{17}$ The Cochrane review ${ }^{13}$ which covers publications until 2012 included another five studies from Germany, three of them were trials being published until 2000 and two trials being published after 2006 (one of these was a multicentre study with one of seven centres being located in Germany). Most of these studies had some methodological shortcomings such as not adjusting for the clustering of data, which results from the specific characteristics of the setting with delivery of the intervention to complete classes, and schools or classes instead of individuals being the unit of randomisation. Therefore, a lack of rigorous evaluation trials on smoking prevention programmes can be inferred especially for Germany.
The present study aims to contribute to overcome shortcomings in the evidence for the efficacy of schoolbased smoking prevention by presenting results of a cluster-randomised trial from Germany. We report findings on the 6-month follow-up effects of a school-based curriculum named 'Eigenständig werden 5+6' ('Becoming independent $5+6$ ') for students in grades 5 and 6 when they are about 10-12 years old. The overall aim of 'Eigenständig werden $5+6$ ' is the prevention of substance use and abuse by increasing substance-specific skills and general life skills of students. Findings presented refer to effects of programme participation on smoking-related knowledge, attitudes towards smoking, perceived norms of smoking, self-efficacy to resist cigarette offers and actual smoking behaviour 6 months after the implementation over 2 school-years has ended, that is, on an average, 26 months after baseline.

\section{METHODS \\ Intervention}

'Eigenständig werden $5+6$ ' is a school-based prevention programme for students in grades 5 and 6 which takes quality criteria of effective prevention programmes into account. ${ }^{8} 91819$ Based on the life skills approach and on the social influence model, 'Eigenständig werden 5+6' aims primarily at the prevention of substance use (ie, tobacco smoking and drinking alcohol) by increasing general life skills as well as substance-specific skills (ie, coping with emotions, stress, problems, pressures to smoke and drink alcohol, increase of refusal skills and decrease of susceptibility to prosmoking and proalcohol social influences).

The programme delivers the contents in 14 units (most of them lasting $90 \mathrm{~min}$ ) and in two workshops (4-6 class-hours) that are evenly distributed over the grades 5 and 6. In detail, each lesson covers a specific topic, for instance, problem-solving, critical thinking, effective communication skills, decision-making, interpersonal relationship skills, self-awareness building skills, empathy, coping with stress and emotions as well as the student's ability to work in a group. The two workshops include several activities about smoking cigarettes and alcohol misuse, and are designed as a student's course with nine challenging stations that address either different aspects of smoking cigarettes or aspects of alcohol misuse. Stations concerning the first workshop include smoking cigarettes, cover risks and disadvantages of smoking, smoking-related knowledge, perceived norms and self-efficacy to refuse cigarette offers as well as strategies of advertisement and industry. The stations of the second workshop deal with the same issues, but tailored to the topic of alcohol misuse. All components are realised by trained teachers within daily school-routine. Further details on the programme have been described elsewhere. ${ }^{20}$

\section{Design}

A five-wave cluster randomised controlled trial with two arms (intervention and control condition) was 
conducted in four German federal states (Bremen, Hesse, North Rhine-Westphalia and Schleswig-Holstein) to evaluate the effects of 'Eigenständig werden $5+6$ '. The randomisation occurred at school level to avoid information exchange between conditions in the school. For randomisation, schools were stratified according to the following criteria: (1) study region, (2) type of school and (3) the number of fifth grade classes per school. According to these strata, schools were randomly assigned to the two arms of the study with a $50 \%$ chance of being allocated to either group by using coin toss method. Intervention group took part in 'Eigenständig werden $5+6$ ' and is compared to control group receiving education as usual, that is, lessons and subjects following the standard school curriculum without specific interventions to foster life skills or to prevent smoking. Baseline assessment took place prior to the intervention in October/November 2010, post-tests were realised in June/July 2011 and June/July 2012, a 6-month follow-up was conducted in December 2012 and a 15-month follow-up was conducted in September/October 2013. For further details, see ref. 20.

\section{Study sample}

A priori sample size determination assuming a significance level of $\alpha=0.05$, power $=0.80$, a $15 \%$ prevention effect, an average cluster size of 20 students per class, an intraclass correlation of 0.02 and a dropout rate of $25 \%$ revealed a recommended sample size of 158 classes and 3160 students at baseline (for detailed description of sample size determination see ref. 20). To recruit the sample, complete lists of all secondary schools of selected regions were obtained from the Ministries of Education of each federal state. An overall of 450 secondary schools in the study regions were invited to participate in the study, of which $323(71.5 \%)$ did express neither approval nor disapproval (figure 1). The decision to participate in the study was expressed by 48 schools (11\%) with 191 classes and 4772 students. Of these 48 schools agreeing to participate, 26 schools with 97 classes and 2437 students were allocated to the intervention group whereas 22 schools with 94 classes and 2335 students were assigned to the control condition. After randomisation, three schools of the intervention group withdrew their consent as well as four teachers of intervention classes refused to take part.

Consequently, baseline data comprise 45 schools, 172 classes and 3444 students with a mean age of 10.37 years $(\mathrm{SD}=0.59) ; 47.9 \%$ girls; with 1685 students in 81 classes in the intervention and 1759 students in 91 classes in the control condition. With respect to different outcome and covariate characteristics, intervention and control students were extensively comparable with the exception of a higher proportion of students of Gymnasiums in the control condition (for detailed baseline sample description see ref. 20). Data presented refer to the baseline (October 2010) and a 6-month follow-up survey (December 2012). It was possible to merge follow-up data of 1255 students in the intervention arm and 1438 students in the control arm (total $\mathrm{N}=2693,78.2 \%$ of $\mathrm{N}=3444$ ) to baseline data. For analysis, datasets with inconsistencies concerning age, gender and smoking status were excluded $(\mathrm{N}=180)$. The final analysis sample consists of 2513 datasets (intervention group: $\mathrm{N}=1179$, control group: $\mathrm{N}=1334)$. The mean age at follow-up was 12.50 years $(\mathrm{SD}=0.58)$.

\section{Measures}

Data were collected through self-completed anonymous questionnaires by teachers and students. The development of the questionnaire and a complete list of variables and constructs assessed in the trial have been documented..$^{20}$ Analyses presented rely on the following outcome measures: lifetime tobacco smoking experience was assessed by asking how many cigarettes have ever been smoked in life. Nine answering categories ('none', 'only a few puffs', '1 cigarette', '2 cigarettes', '3-4 cigarettes', '5-9 cigarettes', '10-19 cigarettes', '20-100 cigarettes' and '>100 cigarettes') were provided. Students having smoked at least a few puffs were considered as ever-smokers. Smoking incidence at the follow-up among baseline never-smokers was determined by combining the answers to lifetime smoking at baseline and follow-up: those who had never smoked at baseline, and indicated any smoking by the follow-up survey, even just a few puffs, were considered as having initiated smoking during the observation period. Current smoking frequency was measured by asking how often the subject currently smokes. Respondents could answer 'I don't smoke,' 'less than once a month', 'at least once a month, but not weekly', 'at least once a week, but not daily' or 'daily'. Responses were dichotomised into students not smoking and students indicating any kind of current smoking.

To assess attitudes towards smoking and risk perception, respondents should rate 11 statements (eg, 'Non-smokers are fitter', 'Non-smokers are cooler', 'If I smoke during the next month, I will get in trouble with my friends'). Answer categories comprise $0=$ 'not true', $1=$ 'somewhat true', $2=$ 'rather true' and $3=$ "totally true', that is, higher values represent a more negative attitude towards smoking and more sensitive perception of risk. Cronbach' $\alpha$ of this scale was 0.80 . A change in attitude from baseline to follow-up was determined by subtracting the baseline value from the follow-up value for each respondent. Smoking-related knowledge was tested through seven statements (eg, 'Cigarettes contain arsenic which is also found in rat poison.') to which respondents could either agree by answering 'true' or disagree by stating 'wrong' or state 'I don't know'. As an indicator of knowledge, the percentage of correct classifications of statements was determined. An increase in knowledge from baseline to follow-up was determined by subtracting the baseline value from the follow-up value for each respondent. Normative expectations were measured on an 11-point scale ranging from $0=$ 'nobody' to 


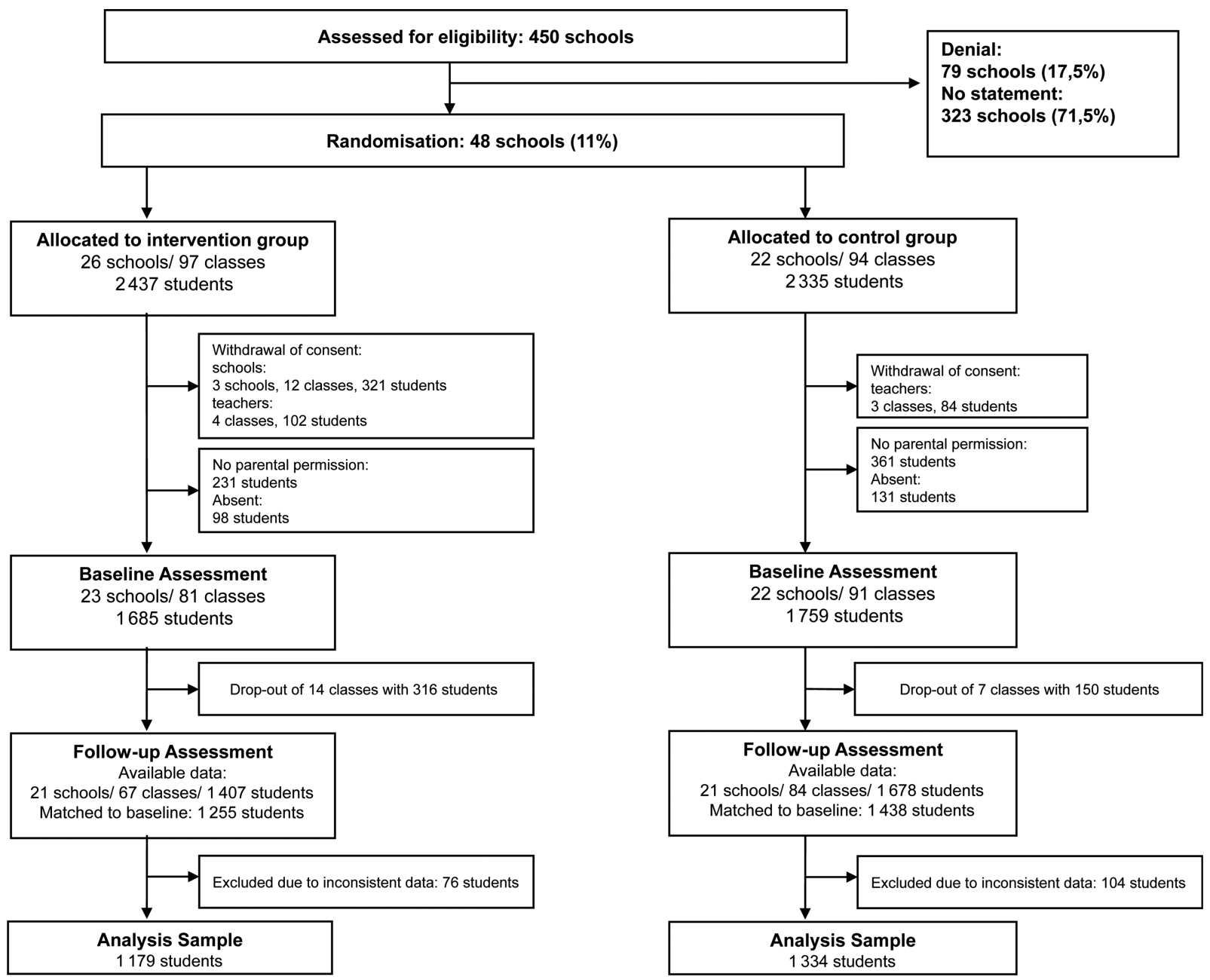

Figure 1 Participation flow chart.

$10=$ 'everybody' on which students had to estimate how many (a) 'Adults in Germany' and (b) 'Adolescents at their age' in Germany smoke. Self-efficacy to refuse cigarette offers was assessed by asking 'To what extent do you dare to refuse cigarettes, even if your friends laugh at you or suspend you on that account?' with answer categories ranging from $0=$ 'not at all' to $3=$ "totally'. Normative expectations and self-efficacy were analysed on single-item level.

Covariate measures were derived from studies that focused on risk factors of adolescent tobacco use, to control for confounding variables that would be theoretically related to the smoking measures. ${ }^{21-23}$ Sociodemographics include age, gender, type of school (gymnasium vs other type of school), migration background (mother and/or father were born outside Germany) and socioeconomic status (Family Affluence Scale $\left.^{24}\right)$. As personal characteristics, rebelliousness and sensation-seeking were assessed with two items in each case. ${ }^{25}$ Parent, sibling and peer smoking were assessed as factors from social environment (no vs any parent/ sibling/peer smoking). Finally, students were asked whether they had participated in a comprehensive prevention programme in elementary school with examples of most broadly disseminated programmes given.

\section{Procedure}

In schools having agreed to participate, teachers of the respective fifth grades, collected the parental informed consent. Students with refusal were excluded from all assessments. Data assessment was conducted in the class room and lasted for $45 \mathrm{~min}$. Research staff was responsible for the distribution, help in completion and collection of the questionnaire. To permit a linking of individual baseline and follow-up data while assuring anonymity, students generated a seven-digit individual code. This procedure has been tested and used in several studies and therefore been inspected and approved by Ethics Committee, data protection and Ministries of Education repeatedly. ${ }^{27}$ Teachers were not involved in the data assessment. At the end of the assessment, all questionnaires were placed in an envelope which was sealed in front of the class. Therefore, every student was assured that neither teachers nor parents were able to see the completed questionnaire. 


\section{Analyses}

Predictors of attrition were examined by multilevel mixed effects logistic regressions. To test for selective attrition, interaction effects for group condition and dependent variable were tested. Descriptive statistics (percentages, means, SDs) are crude values. To analyse the effects of the intervention on smoking behaviour as well as on attitudes and knowledge, multilevel mixed effects regression models using Stata mixed and meqrlogit command were conducted, adjusting for covariates. In case of dichotomous outcomes, effect sizes (Cohen's d) were determined by using the converting formula proposed by Borenstein et al. ${ }^{28}$ Effect sizes for metric outcomes were calculated as proposed by Feingold. ${ }^{29}$ All data analyses were conducted with Stata V.13. ${ }^{30}$

\section{RESULTS}

\section{Attrition analysis}

Among the 3444 students assessed at baseline, there were no data available in the analysis sample for $931 \mathrm{stu}-$ dents $(27 \%)$. Overall, significantly higher attrition was found for male students (31.2\%; $\mathrm{p}<0.001)$, students with a migration background $(36.5 \% ; \mathrm{p}<0.001)$, students indicating current smoking $(45.7 \%$; $\mathrm{p}=0.047)$ or current use of alcohol $(39.8 \% ; \mathrm{p}<0.001)$ at baseline. Furthermore, students dropping out of the study were older than students in the retention sample $\left(\mathrm{M}_{\text {attrition }}=10.5\right.$ vs $\mathrm{M}_{\text {retention }}=10.3$ years of age at baseline; $\mathrm{p}=0.003$ ). Besides these overall effects, no hints for selective attrition were found, that is, the associations between variables listed above and attrition did not differ systematically between the intervention and control group.

\section{Effects on smoking behaviour}

Students in the intervention and control group did not differ in rates for lifetime and current smoking at baseline. At the follow-up, $16 \%$ of intervention students indicated that they had ever smoked in their lives compared with $20.2 \%$ in the control students (figure 2). This difference was statistically significant also after controlling for confounding factors (adjusted $\mathrm{OR}=0.63$; $95 \%$ CI 0.41 to $0.96 ; \mathrm{p}=0.026$ ).

While about $1 \%$ of intervention and control students reported current smoking at baseline, these rates increased to more than $5 \%$ at follow-up in intervention and control groups. Therefore, no group differences could be found in the frequency of current smoking.

At baseline, $95.6 \%$ of the sample were never-smokers ( $\mathrm{N}=2403$; intervention group: $96.4 \%$, control group: 94.9\%). Among these baseline never-smokers, $15.5 \%$ initiated smoking during the observation period. The incidence rate was significantly lower in intervention students, among whom $13.7 \%$ reported the first use of cigarettes between baseline and follow-up, compared with the control group with an incidence rate of $17.1 \%$ (adjusted $\mathrm{OR}=0.66,95 \%$ CI 0.43 to $1.00, \mathrm{p}=0.047$ ).

\section{Effects on smoking-related knowledge and attitudes}

Students of both groups showed about the same level of smoking-related knowledge at baseline with correct answering rates of about $30 \%$ (table 1). At follow-up, students in both groups scored better in the knowledge test: intervention students had a mean percentage of correct answers of 46.75 , while the control students rated, on an average, $37.74 \%$ of the statements correctly. The increase in knowledge was significantly higher in the intervention group (adjusted $\beta=9.38 ; 95 \%$ CI 6.73 to 12.04; $\mathrm{p}<0.001)$.

A comparable pattern was found for attitudes towards smoking: at baseline, both groups did not differ in their attitudes and showed a rather critical perception of smoking with a mean score of 2.04 on the scale ranging from 0 to 3 (table 1). At follow-up, students rated smoking even more negatively with a mean score of 2.24 in the intervention and 2.16 in the control group.
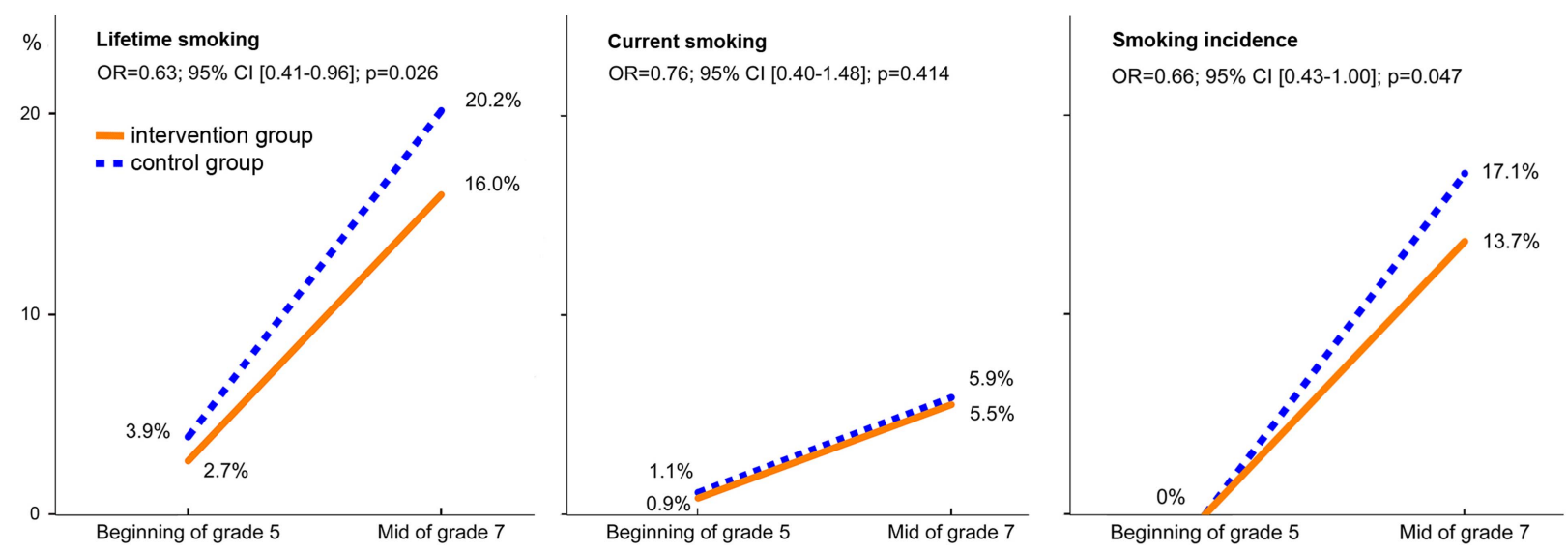

Figure 2 Percentage of lifetime smoking, current smoking at baseline and 6-month follow-up, percentage of smoking incidence at 6-month follow-up among baseline never-smokers and multilevel mixed effects logistic regressions; logistic regressions were adjusted for age, gender, type of school, socioeconomic status, migration background, peer/parent/sibling smoking, sensation seeking, rebelliousness, earlier participation in a prevention programme. 


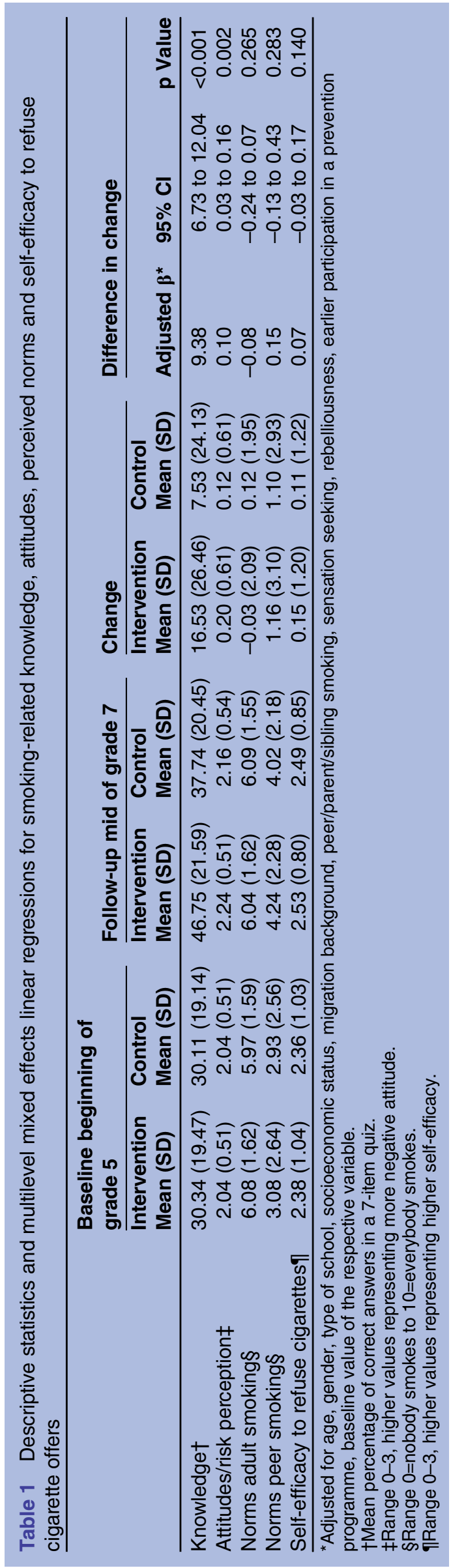

Compared with the control group, this change in attitudes was more pronounced in the intervention group (adjusted $\beta=0.10 ; 95 \%$ CI 0.03 to $0.16 ; p=0.002$ ).

Normative expectations were hardly influenced by the intervention: While intervention students estimated smoking prevalence in adults marginally lower at follow-up compared with their estimation at baseline, a small increase in this estimate could be observed in control students (table 1). For peer smoking, both groups showed a small increase in perceived frequency of smoking. None of these changes turned out to be statistically significant after covariates and baseline estimates were controlled for.

Already at baseline, students in both groups considered themselves rather capable to refuse cigarette offers (table 1). During the observation period, self-efficacy even grew with a slightly higher-but statistically insignificant-increase in intervention students compared with control students.

Effect sizes for significant programme effects were small to medium with $d=0.26$ (lifetime smoking), $d=0.23$ (incidence of smoking), $\mathrm{d}=0.45$ (smoking-related knowledge) and $\mathrm{d}=0.15$ (attitudes/risk perception).

\section{Sensitivity analyses}

Since a substantial proportion of cases was excluded from the analysis sample due to inconsistent data $(\mathrm{N}=180)$ or as they were lost to follow-up $(\mathrm{N}=931)$, we conducted some sensitivity analyses to estimate the risk that the findings might be biased by these exclusions. First, we reran the analyses with inconsistent cases left in the dataset. This reinclusion of inconsistent cases hardly changed the results for metric outcomes with significant effects for knowledge and attitudes and slightly changed results for smoking behaviour with marginally significant associations for lifetime smoking $(\mathrm{p}=0.086)$ and incidence $(p=0.083)$. To address the restriction of sample to complete cases, predictors for higher attrition were used as a guide to create subsamples. As the attrition rate was highest in baseline current smokers, and therefore this (small) subgroup of students smoking already very early might differ substantially from the majority of nonsmoking students, we excluded baseline current smokers $(\mathrm{N}=25)$ completely. For this subsample, we found the same significant effects for lifetime smoking $(\mathrm{p}=0.030)$, knowledge $(p<0.001)$ and attitudes $(p=0.002)$ and no effects on current smoking, norms and self-efficacy. Further factors being associated with a higher risk of attrition (male gender, higher age, migration background) were explored by analysing subsamples of male students, students aged $\geq 11$ years at baseline and students with migration background. In these subsamples, incidence rates in control students exceeded those of intervention by $4-7 \%$ points (reaching significance for older students, $p=0.026$, marginal significance for students with migration background, $\mathrm{p}=0.061$, and failing to reach significance for male students, $\mathrm{p}=0.225$ ). 


\section{DISCUSSION}

The current study aimed to investigate the effects of a school-based prevention programme on smoking-related knowledge, attitudes and behaviour 6 months after the end of programme implementation. Using a clusterrandomised controlled design, findings revealed that the programme participation seems to reduce the prevalence and incidence of lifetime smoking, increase smoking-related knowledge and influence attitudes and perceived risks of smoking towards a more critical perception. Programme effects on the prevalence of current smoking, normative expectations or refusal skills could not be determined. Using Cohen's classification of effect sizes, ${ }^{31}$ effect size was small for smoking behaviour and attitudes, while there was a medium effect on smoking-related knowledge.

The pattern found for effects on smoking behaviour with significant small effects on smoking incidence $(\mathrm{d}=0.23)$ and lifetime smoking $(\mathrm{d}=0.26)$, and no effect on current smoking, can be aligned quite well with the results presented in former meta-analyses: The pooled OR of 0.88 for smoking onset at the longest follow-up revealed by the overall analysis of Thomas et $a l^{13}$ corresponds to an effect size of $d=0.07$, while their subgroup analyses for different intervention approaches provided even moderate effect sizes (ORs between 0.49 and 0.52 corresponding to ds between 0.36 and 0.39). Earlier meta-analyses on school-based drug prevention programmes, ${ }^{14}$ psychosocial smoking prevention programmes $^{15}$ or studies on Project DARE (Drug Abuse Resistance Education), ${ }^{16}$ the most widely used schoolbased drug prevention programme in the USA and focusing on teaching skills to recognise and resist social pressure also determined small programme effects on substance use with d's ranging between 0.08 and 0.18. Finding no effect on current smoking might also be explained to some extent by the young age of the sample with very few students smoking already on a regular basis.

The participation in 'Eigenständig werden 5+6' significantly increased smoking-related knowledge with an effect size of $d=0.45$ - the highest effect found in our analyses. This medium effect is in line with effect sizes ranging from 0.36 to 0.42 determined in meta-analyses referred above. ${ }^{14-16}$ Attitudes were influenced also significantly by the intervention; the small effect $(d=0.15)$ compares well with the small effects in a range from 0.11 to 0.26 having been reported. ${ }^{14-16}$

We were not able to show an effect of the intervention in correcting perceived norms of smoking or in enhancing self-efficacy to refuse offered cigarettes. Students of intervention and control group considered themselves to be rather highly self-efficacious to resist to cigarette offers already at the outset of the study and this rating even increased in both groups at follow-up, that is, a ceiling effect might decrease the discriminative power of the group comparisons. As far as perceived norms of smoking are concerned, a possible explanation of the null-effect is the fact that only a minor part of the intervention (1 subtask among 9 tasks of the smoking workshop at the end of grade 5) deals with the topic of norms. Most of the tasks in the workshop address the facts about and visualisation of the short-term and longterm consequences of smoking; others aim to scrutinise strategies of the tobacco industry or to foster resistance skills.

Some limitations of the current study have to be considered: during the 26-month period from baseline to follow-up, there has been some dropout from the study. The analysis sample $(\mathrm{N}=2513)$ amounted to $73 \%$ of the baseline sample $(\mathrm{N}=3444)$. This dropout was slightly higher than expected in the a priori power calculation $(25 \%)$, but since the baseline sample was somewhat larger than calculated ( $\mathrm{N}=3444$ instead of $\mathrm{N}=3160)$, the analysis sample still exceeds the estimated sample at the follow-up $(75 \%$ of $3160=2370)$. Therefore, the power of the analyses should be adequate. Furthermore, we did not find any hints for selective attrition which could restrict the validity of findings, and sensitivity analyses revealed very comparable patterns of results in subsamples with higher risk for attrition and smoking. The curriculum 'Eigenständig werden 5+6' comprises 14 lessons, $90 \mathrm{~min}$, on an average, and two workshops with 4-6 h of duration which are implemented by teachers in addition to usual education, that is, the programme is quite timeconsuming, it requires some dedication by teachers and it seems unreasonable to assume that the intervention is delivered to all classes exactly in the way and to the extent foreseen by the manual. The analyses in this report were not controlled for treatment integrity, ${ }^{32} 33$ that is, classes with low-treatment fidelity that have only been exposed to parts of the intervention contribute to the same extent to the results of the intervention group as classes with complete programme implementation. Therefore, results can be considered as rather conservative. Another limitation is the reliance on self-reports in the assessment of outcomes. Especially for outcomes assessing smoking behaviour, and also for attitudes towards smoking, answers might be biased by social desirability. These influences might occur more likely in the intervention group since students might be primed by the intervention that smoking is an 'unwanted' behaviour. Nevertheless, since purchase of cigarettes and smoking in public is forbidden by law for minors in Germany, students in the control condition are not free of a social desirability bias as well. For other outcomes like the assessment of refusal skills, validity of self-report data might be limited by restricted accessibility, that is, whether adolescents are really able to judge their own competence to resist cigarette offers. Finally, the study was run in regular public schools of four federal states of Germany, that is, the generalisability might be limited due to these regional constraints and restricted to adolescents visiting regular schools.

On the other hand, some strengths of the current study should be borne in mind: results were derived 
from a cluster-randomised controlled trial, applying sophisticated statistical methods, comprising a range of several outcomes and following students for 6 months after the end of intervention. Therefore, we implemented rather rigorous evaluation methods, which are in line with recommendations like those of the Society for Prevention Research. ${ }^{34}$

Perspectives and open questions for future research might be the following: in the current trial, a further follow-up at 15 months after end of intervention was conducted to explore stability of effects in the longer term. Furthermore, exploring effects on other outcomes than smoking, for example, alcohol use or life skills, is owing for the current study. On the basis of findings for efficacy, effectiveness in relation to costs as well as generalisability to other populations should be investigated. Finally, besides stating whether an intervention is effective or not, mechanisms by which shown effects can be explained theoretically and empirically are to be explored. These mediation analyses may contribute to investigate causal models for preventive intervention, for example, to explore whether knowledge or change in attitude are necessary preconditions for behaviour change. ${ }^{14}$

Acknowledgements Marianne Bayrhuber contributed to the completion of the project by organising the data assessments. Maja Hornberger, Gudrun Wiborg, Birte Walther, Hinnerk Frahm and Heike Kühl-Frese were involved in the development of the prevention programme. Malin Bachmann, Tina Basner, Jana Blumkowski, Dorothea Breckwoldt, Roxana Buzila, Svenja Cleve, Malte Dincher, Eva-Maria Eggers, Nadia El Bouhayati, Almut Engelhardt, Sigrid Feierabend, Sarah Göhlert, Lars Grabbe, Judith Hanewinkel, Oksana Herdt, Semiha Idrizovic, Sabiha Izaamriouane, Martin Karsten, Andre Lischick, Stefanie Otte, Serdar Peker, Ines PohImann, Wiebke Pustal, Gesa Sander, Christoph Schlüter, Björn Seiler, Beate Steiber, Alexander Wierzock and Nicole Wilmes assessed the data. The authors would like to thank their partners: Institut für Qualitätsentwicklung an Schulen Schleswig-Holstein, Landesinstitut für Schule Bremen, Krisenhilfe Bochum, Fachstelle für Suchtprävention im Wetteraukreis, Fachstelle für Suchtprävention des Zweckverbandes der Diakonie Bad Hersfeld, Diakonie Herford, Koordinationsstelle Schulische Suchtvorbeugung, Fachstelle für Suchtvorbeugung Dortmund, Hessische Landesstelle für Suchtfragen, Ginko Stiftung für Prävention and all schools that participated in the study.

Contributors BI performed the statistical analysis and interpretation of the data and drafted the manuscript. $\mathrm{JH}$ contributed to the acquisition of the data and participated in drafting the manuscript. KM contributed to the acquisition and management of the data. RH as principle investigator is responsible for the study concept, design and supervision and helped in drafting the manuscript. All authors conducted a critical revision of the article and granted final approval of the article for important intellectual content.

Funding This work was supported by German Cancer Aid (Grant Number: 108374).

\section{Competing interests None.}

Ethics approval Ethics approval was provided by the Ethics Committee of the Medical Faculty of the University of Kiel (Ref.: D 419/10). Additional approvals (eg, from ministries of education and parents) were sought as required.

Provenance and peer review Not commissioned; externally peer reviewed.

Data sharing statement No additional data are available.

Open Access This is an Open Access article distributed in accordance with the Creative Commons Attribution Non Commercial (CC BY-NC 3.0) license, which permits others to distribute, remix, adapt, build upon this work non- commercially, and license their derivative works on different terms, provided the original work is properly cited and the use is non-commercial. See: http:// creativecommons.org/licenses/by-nc/3.0/

\section{REFERENCES}

1. Lim SS, Vos T, Flaxman AD, et al. A comparative risk assessment of burden of disease and injury attributable to 67 risk factors and risk factor clusters in 21 regions, 1990-2010: a systematic analysis for the Global Burden of Disease Study 2010. Lancet 2013;380: 2224-60.

2. Bundeszentrale für gesundheitliche Aufklärung. Der Tabakkonsum Jugendlicher und junger Erwachsener in Deutschland 2012. Ergebnisse einer aktuellen Repräsentativbefragung und Trends. Köln: Bundeszentrale für gesundheitliche Aufklärung, 2013.

3. Robert Koch-Institut. Die Gesundheit von Kindern und Jugendlichen in Deutschland. Berlin: Robert Koch-Institut, 2013.

4. World Health Organisation. Life skills education in schools, parts 1 and 2. Geneva: WHO, Division of Mental Health, 1994.

5. Evans RI. Smoking in children: developing a social psychological strategy of deterrence. Prev Med 1976;51:122-7.

6. Donaldson SI, Graham JW, Hansen WB. Testing the generalizability of intervening mechanism theories: understanding the effects of adolescent drug use prevention interventions. J Behav Med 1994;17:195-216.

7. Flay BR. The promise of long-term effectiveness of school-based smoking prevention programs: a critical review of reviews. Tob Induc Dis 2009;5:7.

8. Cuijpers P. Effective ingredients of school-based drug prevention programs. A systematic review. Addict Behav 2002;27:1009-23.

9. Gottfredson DC, Wilson DB. Characteristics of effective school-based substance abuse prevention. Prev Sci 2003;4:27-38.

10. Flay BR. School-based smoking prevention programs with the promise of long-term effects. Tob Induc Dis 2009;5:6.

11. Skara S, Sussman S. A review of 25 long-term adolescent tobacco and other drug use prevention program evaluations. Prev Med 2003;37:451-74.

12. Wiehe SE, Garrison MM, Christakis DA, et al. A systematic review of school-based smoking prevention trials with long-term follow-up. $J$ Adolesc Health 2005;36:162-9.

13. Thomas RE, McLellan J, Perera R. School-based programmes for preventing smoking. Cochrane Database Syst Rev 2013;4: CD001293.

14. Tobler NS, Stratton HH. Effectiveness of school-based drug prevention programs: a meta-analysis of the research. J Prim Prev 1997;18:71-128.

15. Hwang MS, Yeagley KL, Petosa R. A meta-analysis of adolescent psychosocial smoking prevention programs published between 1978 and 1997 in the United States. Health Educ Behav 2004;31:702-19.

16. Ennett ST, Tobler NS, Ringwalt CL, et al. How effective is drug abuse resistance education? A meta-analysis of Project DARE outcome evaluations. Am J Public Health 1994:84:1394-401.

17. Müller-Riemenschneider F, Rasch A, Bockelbrink A, et al. Effectiveness and cost-effectiveness of behavioural strategies in the prevention of cigarette smoking. GMS Health Technol Assess 2008;4:Doc10.

18. Cuijpers P. Three decades of drug prevention research. Drug Educ Prev Polic 2003;10:7-20.

19. Hansen WB, Dusenbury L, Bishop D, et al. Substance abuse prevention program content: systematizing the classification of what programs target for change. Health Educ Res 2007;22:351-60.

20. Hansen J, Hanewinkel R, Maruska K, et al. The 'Eigenständig werden' prevention trial: a cluster randomised controlled study on a school-based life skills programme to prevent substance use onset. BMJ Open 2011;1:e000352.

21. Gibbons FX, Gerrard M. Predicting young adults' health risk behavior. J Pers Soc Psychol 1995;69:505-17.

22. Hawkins JD, Catalano RF, Miller JY. Risk and protective factors for alcohol and other drug problems in adolescence and early adulthood: implications for substance abuse prevention. Psychol Bull 1992;112:64-105.

23. Petraitis J, Flay BR, Miller TQ. Reviewing theories of adolescent substance use: organizing pieces in the puzzle. Psychol Bull 1995;117:67-86.

24. Currie $\mathrm{C}$, Molcho $\mathrm{M}$, Boyce $\mathrm{W}$, et al. Researching health inequalities in adolescents: the development of the Health Behaviour in School-Aged Children (HBSC) family affluence scale. Soc Sci Med 2008;66:1429-36 
25. Stephenson MT, Hoyle RH, Palmgreen P, et al. Brief measures of sensation seeking for screening and large-scale surveys. Drug Alcohol Depend 2003;72:279-86.

26. Burt RD, Dinh KT, Peterson AV Jr, et al. Predicting adolescent smoking: a prospective study of personality variables. Prev Med 2000;30:115-25.

27. Galanti MR, Siliquini R, Cuomo L, et al. Testing anonymous link procedures for follow-up of adolescents in a school-based trial: the EU-DAP pilot study. Prev Med 2007;44:174-7.

28. Borenstein M, Hedges LV, Higgins JPT, et al. Introduction to meta-analysis. Chichester: Jon Wiley \& Sons, 2009.

29. Feingold A. Effect sizes for growth-modeling analysis for controlled clinical trials in the same metric as for classical analysis. Psychol Methods 2009;14:43-53.
30. StataCorp. Stata Statistical Software: Release 13. College Stations, Texas: StataCorp, 2013.

31. Cohen J. Statistical power analyses for behavioral science. Hilldale, New York: Erlbaum, 1988.

32. Bishop DC, Pankratz MM, Hansen WB, et al. Measuring fidelity and adaptation: reliability of a instrument for school-based prevention programs. Eval Health Prof. Epub ahead of print: 19 Feb 2013.

33. Dusenbury L, Brannigan $\mathrm{R}$, Falco $\mathrm{M}$, et al. A review of research on fidelity of implementation: implications for drug abuse prevention in school settings. Health Educ Res 2003;18:237-56.

34. Flay BR, Biglan A, Boruch RF, et al. Standards of evidence: criteria for efficacy, effectiveness and dissemination. Prev $\mathrm{S} c i$ 2005;6:151-75. 